\title{
SPECTRAL ANISOTROPY OF ELSÄSSER VARIABLES IN TWO-DIMENSIONAL WAVE-VECTOR SPACE AS OBSERVED IN THE FAST SOLAR WIND TURBULENCE
}

\author{
Limei Yan ${ }^{1}$, Jiansen He ${ }^{1,2,5}$, Lei Zhang ${ }^{1,2}$, Chuanyi Tu ${ }^{1}$, Eckart Marsch ${ }^{2}$, Christopher H. K. Chen ${ }^{3}$, \\ Xin WANG ${ }^{1}$, LinghuA WANG ${ }^{1}$, AND Robert T. Wicks ${ }^{4}$ \\ ${ }^{1}$ School of Earth and Space Sciences, Peking University, 100871 Beijing, China \\ ${ }^{2}$ State Key Laboratory of Space Weather, Chinese Academy of Sciences, Beijing 100190, China; jshept@gmail.com \\ ${ }^{3}$ Institute for Experimental and Applied Physics, Christian Albrechts University at Kiel, D-24118 Kiel, Germany \\ ${ }^{4}$ Department of Physics, Imperial College London, London, SW7 2AZ, UK \\ ${ }^{5}$ Department of Space and Climate Physics, University College London, Gower Street, London, UK \\ Received 2015 September 1; accepted 2015 December 7; published 2016 January 12
}

\begin{abstract}
Intensive studies have been conducted to understand the anisotropy of solar wind turbulence. However, the anisotropy of Elsässer variables $\left(\boldsymbol{Z}^{ \pm}\right)$in $2 \mathrm{D}$ wave-vector space has yet to be investigated. Here we first verify the transformation based on the projection-slice theorem between the power spectral density $\operatorname{PSD}_{2 \mathrm{D}}\left(k_{\|}, k_{\perp}\right)$ and the spatial correlation function $\mathrm{CF}_{2 \mathrm{D}}\left(r_{\|}, r_{\perp}\right)$. Based on the application of the transformation to the magnetic field and the particle measurements from the WIND spacecraft, we investigate the spectral anisotropy of Elsässer variables $\left(\boldsymbol{Z}^{ \pm}\right)$, and the distribution of residual energy $E_{\mathrm{R}}$, Alfvén ratio $R_{\mathrm{A}}$, and Elsässer ratio $R_{\mathrm{E}}$ in the $\left(k_{\|}, k_{\perp}\right)$ space. The spectra $\operatorname{PSD}_{2 \mathrm{D}}\left(k_{\|}, k_{\perp}\right)$ of $\boldsymbol{B}, \boldsymbol{V}$, and $\boldsymbol{Z}_{\text {major }}$ (the larger of $\boldsymbol{Z}^{ \pm}$) show a similar pattern that $\operatorname{PSD}_{2 \mathrm{D}}\left(k_{\|}, k_{\perp}\right)$ is mainly distributed along a ridge inclined toward the $k_{\perp}$ axis. This is probably the signature of the oblique Alfvénic fluctuations propagating outwardly. Unlike those of $\boldsymbol{B}, \boldsymbol{V}$, and $\boldsymbol{Z}_{\text {major }}$, the spectrum $\operatorname{PSD}_{2 \mathrm{D}}\left(k_{\|}, k_{\perp}\right)$ of $\boldsymbol{Z}_{\text {minor }}$ is distributed mainly along the $k_{\perp}$ axis. Close to the $k_{\perp}$ axis, $\left|E_{\mathrm{R}}\right|$ becomes larger while $R_{\mathrm{A}}$ becomes smaller, suggesting that the dominance of magnetic energy over kinetic energy becomes more significant at small $k_{\|}$. $R_{\mathrm{E}}$ is larger at small $k_{\|}$, implying that $\operatorname{PSD}_{2 \mathrm{D}}\left(k_{\|}, k_{\perp}\right)$ of $\boldsymbol{Z}_{\text {minor }}$ is more concentrated along the $k_{\perp}$ direction as compared to that of $Z_{\text {major }}$. The residual energy condensate at small $k_{\|}$is consistent with simulation results in which $E_{\mathrm{R}}$ is spontaneously generated by Alfvén wave interaction.
\end{abstract}

Key words: solar wind - turbulence - waves

\section{INTRODUCTION}

Magnetohydrodynamic (MHD) turbulence in the solar wind is considered to evolve a cascade of energy over different scales caused by the nonlinear interaction between counterpropagating Alfvén waves, which has been studied in detail by asymptotic solution (Howes \& Nielson 2013) and numerical simulation (Nielson et al. 2013). The cascade is anisotropic with the cascading direction mainly perpendicular to the local mean magnetic field (e.g., Goldreich \& Sridhar 1995). When the oppositely directed Alfvén waves carry unequal energy, the turbulence is imbalanced. Imbalanced weak (Galtier et al. 2000; Lithwick \& Goldreich 2003) and strong (Lithwick et al. 2007) turbulence have been studied intensively. In some theoretical studies, the energy spectrum of the Elsässer variables $\left(\boldsymbol{Z}^{ \pm}=\boldsymbol{V} \pm \boldsymbol{b}, \boldsymbol{b}=\frac{\boldsymbol{B}}{\sqrt{\mu_{0} \rho}}\right) E^{ \pm}$have the same scaling with different amplitudes. The scaling is $E^{+} \propto E^{-} \propto k_{\perp}^{-3 / 2}$ with the phenomenon of dynamic alignment (Perez \& Boldyrev 2009), and $E^{+} \propto E^{-} \propto k_{\perp}^{-5 / 3}$ without the phenomenon of dynamic alignment (Lithwick et al. 2007). In the solar wind, especially in fast streams, imbalanced turbulence is usually observed (one of $\boldsymbol{Z}^{ \pm}$is dominating). We define the dominant mode as $\boldsymbol{Z}_{\text {major }}$, which is typically the Alfvén wave propagating outward from the Sun, while the nondominant mode $\boldsymbol{Z}_{\text {minor }}$ is weak and complicated. The nondominant mode has been suggested to be the inward propagating Alfvén wave at high frequencies and to be compressive events at low

\footnotetext{
5 To whom any correspondence should be addressed.
}

frequencies (e.g., Bruno et al. 1996), or magnetic structures (Tu \& Marsch 1992, 1993).

Without temperature anisotropies and relative drifts, if the MHD turbulence is only composed of counterpropagating Alfvén waves without nonlinear interaction, the residual energy $E_{\mathrm{R}}=v^{2}-b^{2}$ would be zero. However in the solar wind, outward propagating Alfvén waves are often observed, while inward propagating Alfvén waves are rarely observed. Besides, there are also many structures like tangential discontinuities in the solar wind that may contribute more to magnetic disturbances than the velocity fluctuations. These factors would lead to the residual energy being nonzero. In the solar wind turbulence, the residual energy at small scales near the dissipation range is usually less than 0 (e.g., Belcher \& Davis 1971; Matthaeus \& Goldstein 1982; Boldyrev et al. 2012; Chen et al. 2013). This is also noted in simulations (e.g., Grappin et al. 1983; Müller \& Biskamp 2005; Gogoberidze et al. 2012). The residual energy exhibits a power-law behavior in the inertial range with a spectral slope of -2 , as revealed from observation (Chen et al. 2012) and simulation (Boldyrev et al. 2011; Franci et al. 2015). In recent simulations, the residual energy is concentrated at small $k_{\|}$(Boldyrev \& Perez 2009; Wang et al. 2011), which is probably spontaneously generated by interacting Alfvén waves (Wang et al. 2011). Bavassano et al. (1998), Roberts et al. (1987), and Wicks et al. (2011) showed a high residual energy at a low frequency/small $k$ from observation, however, they have not investigated whether this is parallel to the magnetic field. The distribution of the residual energy in the wave-vector space will allow us to compare with these simulation results. 
The presence of a mean magnetic field may lead to spectral anisotropy of MHD turbulence (Shebalin et al. 1983). Goldreich \& Sridhar (1995) investigated the anisotropy in a balanced strong MHD turbulence with vanishing cross-helicity, revealing a spectrum perpendicular to the magnetic field of $\mathrm{E}\left(k_{\perp}\right) \sim k_{\perp}^{-5 / 3}$, a parallel spectrum $\mathrm{E}\left(k_{\|}\right) \sim k_{\|}^{-2}$, and a scaling relation $k_{\|} \sim k_{\perp}^{2 / 3}$ based on the critical balance assumption, i.e., linear wave periods are comparable to the nonlinear turnover timescales. The anisotropic power and scaling of magnetic field fluctuations in the inertial range of high-speed solar wind turbulence was first reported by Horbury et al. (2008), who introduced the method to estimate the scale-dependent local $\boldsymbol{B}_{0}$. The reduced spectrum has an index near -2 when $\theta_{B V} \rightarrow 0$ and an index near $-5 / 3$ when $\theta_{B V} \rightarrow 90$ where $\theta_{B V}$ is the angle between the magnetic field and the flow. Podesta (2009) gave similar results using magnetic field measurements from STEREO. Luo \& Wu (2010) and Chen et al. (2011) also found a similar conclusion for the magnetic structure function. When the second-order structure function of the magnetic fluctuations is decomposed into components perpendicular $\left(\delta B_{\perp}^{2}\right)$ and parallel $\left(\delta B_{\|}^{2}\right)$ to the mean field, both components show spectral index anisotropy between the ion and electron gyroscales in the fast solar wind (Chen et al. 2010). At these small scales the spectral index of $\delta B_{\perp}^{2}$ is -2.6 at large angles and -3 or steeper at small angles. This kind of spectral anisotropy of solar wind turbulence in the inertial range is probably related to the intermittency (Wang et al. 2014). Wicks et al. (2011) studied the anisotropy of the Elsässer variables in fast solar wind based on the reduced spectrum, finding that the dominant Elsässer mode is isotropic at low frequencies but becomes increasingly anisotropic at higher frequencies, while the nondominant mode is anisotropic throughout.

The spectral anisotropy has been studied extensively based on the reduced spectrum, while the anisotropy in wave-vector space is relatively rarely studied. The $K$-filtering method has been applied to the Cluster observations to investigate the anisotropy in wave-vector space (e.g., Narita et al. 2010; Sahraoui et al. 2010). However, this method is sensitive only to a limited number of wave modes and the scales comparable to the inter-spacecraft distance (Horbury et al. 2012). Based on single spacecraft measurements, $\mathrm{He}$ et al. (2013) first constructed the normalized power spectral density (PSD) of magnetic field fluctuations $(\boldsymbol{B})$ in 2D wave-vector space. They found that the PSD of $\boldsymbol{B}$ shows an anisotropic distribution, which is mainly characterized by a ridge distribution inclined more toward $k_{\perp}$ as compared to $k_{\|}$. The spectral anisotropy of velocity and Elsässer variables in wave-vector space has not been previously investigated. We will study them in this paper using the method contributed by $\mathrm{He}$ et al. (2013). Moreover, we will investigate the distribution of residual energy $E_{\mathrm{R}}=E_{v}-E_{b}$, Alfvén ratio $R_{\mathrm{A}}=\frac{E_{v}}{E_{b}}$, and Elsässer ratio $R_{\mathrm{E}}=\frac{E_{Z_{\text {minor }}}}{E_{Z_{\text {major }}}}$ in the wave-vector space.

\section{BENCHMARK TEST OF THE CONVERSION BETWEEN $\mathrm{CF}_{2 \mathrm{D}}$ AND PSD $2 \mathrm{D}$}

To test the conversion between $\mathrm{CF}_{2 \mathrm{D}}$ and $\mathrm{PSD}_{2 \mathrm{D}}$ based on the projection-slice theorem, we first assume a double Gaussian distribution, a strong parallel component and a weak perpendicular component, for $\mathrm{CF}_{2 \mathrm{D}}$ using the formula given below:

$$
\begin{aligned}
& \mathrm{CF}_{2 \mathrm{D}}\left(r_{\|}, r_{\perp}\right)=\exp \left(-\frac{r_{\|}^{2}}{2 \sigma_{\| 1}^{2}}\right) \cdot \exp \left(-\frac{r_{\perp}^{2}}{2 \sigma_{\perp 1}^{2}}\right) \\
& +3 \cdot \exp \left(-\frac{r_{\|}^{2}}{2 \sigma_{\| 2}^{2}}\right) \cdot \exp \left(-\frac{r_{\perp}^{2}}{2 \sigma_{\perp 2}^{2}}\right) .
\end{aligned}
$$

Based on this assumption, there are three ways to obtain the $\mathrm{PSD}_{2 \mathrm{D}}$. The first way is to get the $\mathrm{PSD}_{2 \mathrm{D}}$ directly from the corresponding formula:

$$
\begin{aligned}
& \operatorname{PSD}_{2 \mathrm{D}}\left(k_{\|}, k_{\perp}\right) \\
& =\sigma_{\| 1} \cdot \exp \left(-\frac{k_{\|}^{2} \cdot \sigma_{\| 1}^{2}}{2}\right) \cdot \sigma_{\perp 1} \cdot \exp \left(-\frac{k_{\perp}^{2} \cdot \sigma_{\perp 1}^{2}}{2}\right) \\
& \quad+3 \cdot \sigma_{\| 2} \cdot \exp \left(-\frac{k_{\|}^{2} \cdot \sigma_{\| 2}^{2}}{2}\right) \cdot \sigma_{\perp 2} \cdot \exp \left(-\frac{k_{\perp}^{2} \cdot \sigma_{\perp 2}^{2}}{2}\right) .
\end{aligned}
$$

The second way is to do the transformation (PSD tomography method) based on the projection-slice theorem. First, we make the one-dimensional integration (1D-INT) of $\mathrm{CF}_{2 \mathrm{D}}$ along the direction $\left(\boldsymbol{u}^{\prime}\right)$ normal to $\boldsymbol{k}$ to get $1 \mathrm{D}-\mathrm{CF}$ at each angle:

$$
\begin{aligned}
& \mathrm{CF}_{1 \mathrm{D}}\left(r, \theta_{k}\right)=\int_{-\infty}^{+\infty} \mathrm{CF}_{2 \mathrm{D}}\left(r \cos \theta_{k}\right. \\
& \left.-u^{\prime} \sin \theta_{k}, r \sin \theta_{k}+u^{\prime} \cos \theta_{k}\right) d u^{\prime} .
\end{aligned}
$$

Second, we calculate the Fourier transformation (FT) of the $1 \mathrm{D}-\mathrm{CF}$ to get the corresponding slice of 2D-PSD at each angle:

$$
\operatorname{PSD}_{2 \mathrm{D}}\left(k, \theta_{k}\right)=\int_{-\infty}^{+\infty} \mathrm{CF}_{1 \mathrm{D}}\left(r, \theta_{k}\right) \exp (-i k r) d r .
$$

Finally, the $\mathrm{PSD}_{2 \mathrm{D}}$ is assembled by putting the slices of $2 \mathrm{D}$ PSD at each angle together. The third way is to do the twodimensional FT (2D-FT) of $\mathrm{CF}_{2 \mathrm{D}}\left(r_{\|}, r_{\perp}\right)$ :

$$
\begin{aligned}
& \operatorname{PSD}_{2 \mathrm{D}}\left(k_{\|}, k_{\perp}\right)=\int_{-\infty}^{+\infty} \int_{-\infty}^{+\infty} \mathrm{CF}_{2 \mathrm{D}}\left(r_{\|}, r_{\perp}\right) \\
& \quad \times \exp \left(-i\left(k_{\|} r_{\|}+k_{\perp} r_{\perp}\right)\right) d r_{\|} d r_{\perp} .
\end{aligned}
$$

Here, we set $\sigma_{\| 1}=0.25, \quad \sigma_{\perp 1}=2.0, \quad \sigma_{\| 2}=2.0$, and $\sigma_{\perp 2}=0.25$. The origin $\mathrm{CF}_{2 \mathrm{D}}$ and the transferred $\mathrm{PSD}_{2 \mathrm{D}}$ obtained by the three methods are given in Figure 1. From Figure 1, the $\mathrm{PSD}_{2 \mathrm{D}}$ obtained by the three different ways are in accordance with each other. This confirms that the PSD tomography method based on the projection-slice theorem is credible.

\section{DATA ANALYSIS AND RESULTS}

Four fast solar wind streams, with their magnetic fields measured by the Magnetic Field Investigation (MFI; Lepping et al. 1995) and particle distribution measured by the ThreeDimensional Plasma Analyser (3DP; Lin et al. 1995), are investigated at a time cadence of $3 \mathrm{~s}$. The time intervals for the four fast solar wind streams are from 12:00 UT 1995 January 30 to 00:00 UT February 4 (stream 1), from 06:00 UT 2007 January 17 to 06:00 UT January 20 (stream 2), from 00:00 UT 2008 February 11 to 12:00 UT February 14 (stream 3), and from 12:00 UT 2008 July 12 to 12:00 UT July 15 (stream 4), 

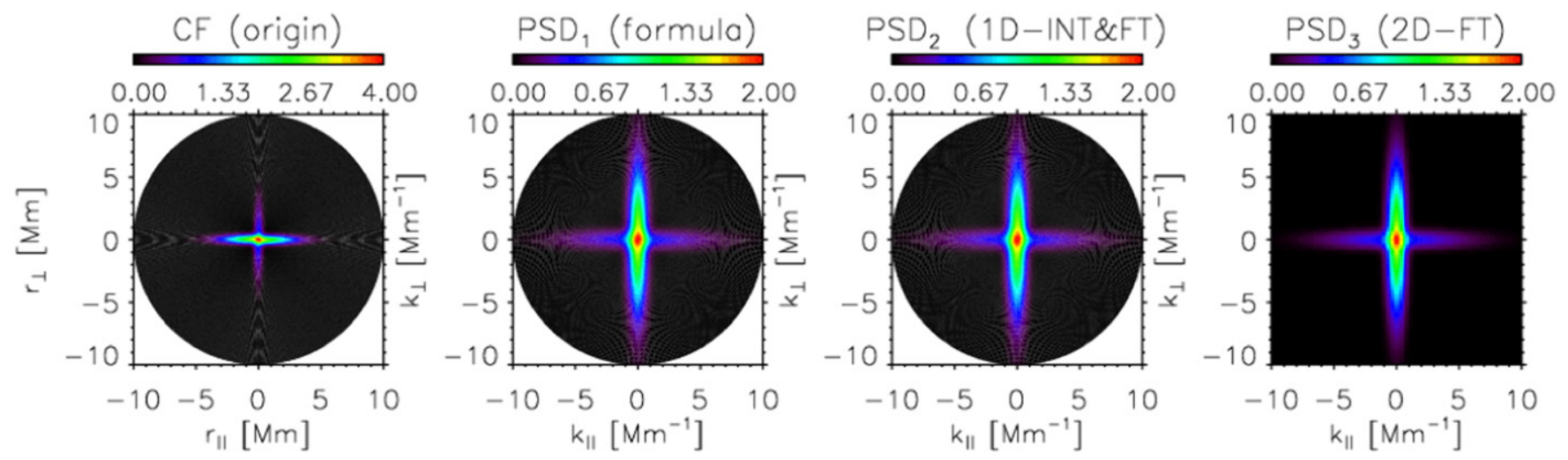

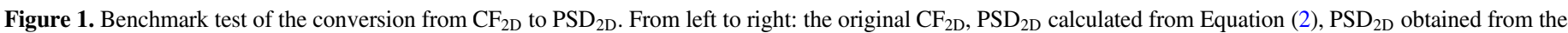
transformation based on the projection theorem that involves $1 \mathrm{D}-\mathrm{INT}$ and FT, and $\mathrm{PSD}_{2 \mathrm{D}}$ obtained by $2 \mathrm{D}-\mathrm{FT}$.

respectively. The four streams are typical fast streams, with a speed more than $600 \mathrm{~km} \mathrm{~s}^{-1}$, a density of $2-4 \mathrm{~cm}^{-3}$, a temperature around $20 \mathrm{eV}$, and a magnetic field of 4-6 nT.

Based on the second-order structure function $\mathrm{SF}(\tau)=\left\langle\left(\boldsymbol{B}\left(t+\frac{\tau}{2}\right)-\boldsymbol{B}\left(t-\frac{\tau}{2}\right)\right)^{2}\right\rangle$, the correlation function $\mathrm{CF}(\tau)=\left\langle\delta \boldsymbol{B}\left(t-\frac{\tau}{2}\right) \cdot \delta \boldsymbol{B}\left(t+\frac{\tau}{2}\right)\right\rangle$, and the angle between the radial direction and local magnetic field, we can get the $2 \mathrm{D}$ correlation function $\mathrm{CF}_{2 \mathrm{D}}$ (see $\mathrm{He}$ et al. 2013 for detailed derivations). Then, the transformation from $\mathrm{CF}_{2 \mathrm{D}}$ to $\mathrm{PSD}_{2 \mathrm{D}}$, based on the projection-slice theorem suggested by $\mathrm{He}$ et al. (2013), is conducted to $\boldsymbol{B}, \boldsymbol{V}, \boldsymbol{Z}_{\text {major }}$, and $\boldsymbol{Z}_{\text {minor }}$ to get $\mathrm{PSD}_{2 \mathrm{D}, B}, \mathrm{PSD}_{2 \mathrm{D}, V}, \mathrm{PSD}_{2 \mathrm{D}, Z_{\text {major }}}$, and $\mathrm{PSD}_{2 \mathrm{D}, Z_{\text {minor }}}$, respectively. This method yields the relative normalized values $\left(\mathrm{PSD}_{2 \mathrm{D} \text {,relative }}\right)$. To get the absolute values, we use the following formula:

$$
\operatorname{PSD}_{2 \mathrm{D}, \text { absolute }}\left(k, \theta_{k}\right)=\operatorname{PSD}_{2 \mathrm{D} \text {, relative }}\left(k, \theta_{k}\right) \cdot \frac{\text { Power }_{\text {absolute }}}{\text { Power }_{\text {relative }}}
$$

with Power $_{\text {absolute }}=\int_{f_{0}}^{f_{1}}$ PSD $_{\mathrm{FFT}} d f$ and Power $_{\text {relative }}=$ $\int_{f_{0}}^{f_{1}} \int_{0}^{2 \pi} \mathrm{PSD}_{2 \mathrm{D} \text {, relative }} \cdot f d f d \theta . f_{0}$ and $f_{1}$ stand for the lower and upper limits of the frequency range used to calculate the power, respectively. Here, $f_{0}$ and $f_{1}$ are set to $10^{-4}$ and $0.067 \mathrm{~Hz}$. $\mathrm{PSD}_{\mathrm{FFT}}$ is obtained by the fast Fourier transformation of the whole time sequence. Then the residual energy $E_{\mathrm{R}}=\mathrm{PSD}_{2 \mathrm{D} \text {, absolute, } \boldsymbol{V}}-\mathrm{PSD}_{2 \mathrm{D}, \text { absolute, } \boldsymbol{b}}, \quad$ Alfvén ratio $R_{\mathrm{A}}=\frac{\mathrm{PSD}_{2 \mathrm{D} \text {,absolute } V}}{\mathrm{PSD}_{2 \mathrm{D} \text {,absolut }, b},}$, and Elsässer ratio $R_{\mathrm{E}}=\frac{\mathrm{PSD}_{2 \mathrm{D} \text {,absolute }, Z_{\text {minor }}}}{\mathrm{PSD}_{2 \mathrm{D} \text {,absolute }, Z_{\text {major }}}}$ in wave-vector space are investigated consequently.

Here we use the scale-dependent local mean magnetic field to define the parallel direction (Horbury et al. 2008). While this introduces higher order correlations into the measurement of the spectrum (Matthaeus et al. 2012), in recent turbulence theories the parallel direction is associated with Alfvén wave packet propagation, for which the relevant direction is the local mean field. It is the local mean field coordinate system that helps us to reveal the presence of two-component kinetic waves, ion-cyclotron waves, and kinetic Alfvén waves, which propagate quasi-parallel and quasi-perpendicularly to the local mean field direction, respectively (He et al. 2011). Here we only consider the same average flow speed for different magnetic field directions. In the future, it would be helpful to include the solar wind speed difference in different magnetic field directions, e.g., larger average speeds when the local mean field direction is perpendicular to the flow than when it is radial, which has been reported by Matteini et al. (2014). Therefore, it is expected that the wave-vector anisotropy would be weaker when adopting the angle-dependent flow speeds, although this would not be a large effect at $1 \mathrm{AU}$.

Figure 2 displays the spectra $\mathrm{PSD}_{2 \mathrm{D}}$ of magnetic field $\boldsymbol{B}$ (upper panels) and velocity $\boldsymbol{V}$ (lower panels) for the four fast streams. The spectra $\mathrm{PSD}_{2 \mathrm{D}}$ of $\boldsymbol{B}$ behave similar to that obtained by $\mathrm{He}$ et al. (2013). The spectra $\mathrm{PSD}_{2 \mathrm{D}}$ of $\boldsymbol{B}$ for the four fast streams show a similar anisotropic distribution in wave-vector space. The PSD is distributed mainly along a ridge that is inclined toward the $k_{\perp}$ axis. Besides the similarity, the distribution of $\mathrm{PSD}_{2 \mathrm{D}}$ also shows some difference between different streams. For example, stream 1 and stream 2 show a component that is aligned with the $k_{\perp}$ axis. We are currently not sure whether the difference between different streams is caused by some underlying physical difference, by the method uncertainty, or by both. In the future, more effort needs to be done to quantitatively estimate the method uncertainty and distinguish it from the physical signal. The $\mathrm{PSD}_{2 \mathrm{D}}$ of $\boldsymbol{V}$ shows a similar anisotropy pattern as that of $\boldsymbol{B}$, suggesting the signature of oblique Alfvén waves.

To investigate the spectral anisotropy of Elsässer variables, the Elsässer spectra in wave-vector space are obtained (Figure 3). The $\mathrm{PSD}_{2 \mathrm{D}}$ of $\boldsymbol{Z}_{\text {major }}$ and $\boldsymbol{Z}_{\text {minor }}$ both show anisotropy in the wave-vector space. However, the anisotropy pattern is different for $\boldsymbol{Z}_{\text {major }}$ and $\boldsymbol{Z}_{\text {minor }}$. The $\mathrm{PSD}_{2 \mathrm{D}}$ of $\boldsymbol{Z}_{\text {major }}$ share a similar anisotropic pattern with that of $\boldsymbol{B}$, and $\mathrm{V}$, while the $\mathrm{PSD}_{2 \mathrm{D}}$ of $\boldsymbol{Z}_{\text {minor }}$ show a very different anisotropy with the main features of $\mathrm{PSD}_{2 \mathrm{D}}$ distributed along the $k_{\perp}$ axis. The $\mathrm{PSD}_{2 \mathrm{D}}$ of $\boldsymbol{Z}_{\text {minor }}$ normalized to the $\mathrm{PSD}_{2 \mathrm{D}}$ with the same $k_{\perp}$, but with $k_{\|}=0$ (upper panels in Figure 4), reveal further evidence that the $\mathrm{PSD}_{2 \mathrm{D}}$ of $\boldsymbol{Z}_{\text {minor }}$ is mainly distributed at small $k_{\|}$. These results suggest that the anisotropy of the nondominant mode $\boldsymbol{Z}_{\text {minor }}$ is stronger than that of the dominant mode $\boldsymbol{Z}_{\text {major }}$, which is consistent with the observational result based on the reduced spectrum (Wicks et al. 2011) and the simulation result (Cho \& Lazarian 2014).

The residual energy $E_{\mathrm{R}}$ for all of the four fast streams is less than 0 , meaning that the magnetic energy is dominant over kinetic energy. The residual energy $E_{\mathrm{R}}$ is normalized to the $k_{\perp}$ axis (lower panels in Figure 4), using the formula $E_{\mathrm{R}, \text { norm }}=\frac{E_{\mathrm{R}}\left(k_{\|}, k_{\perp}\right)}{E_{\mathrm{R}}\left(k_{\|}=0, k_{\perp}\right)}$. As seen from the normalized residual 


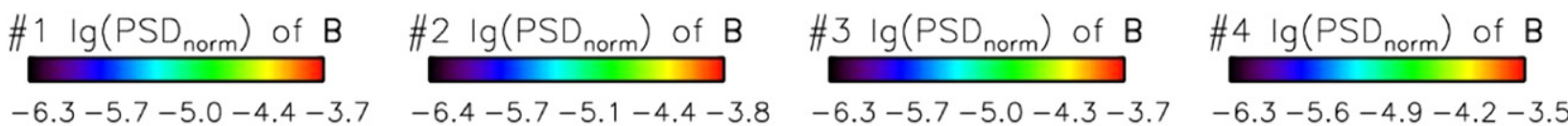

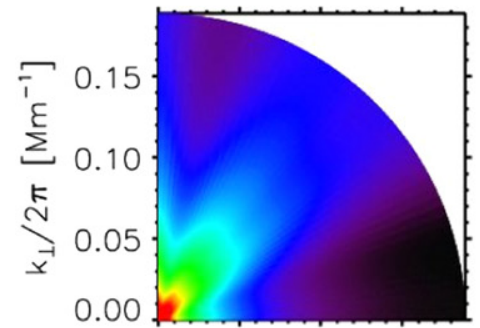

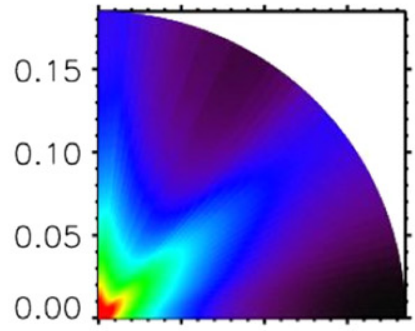

0.000 .050 .100 .15

0.000 .050 .100 .15
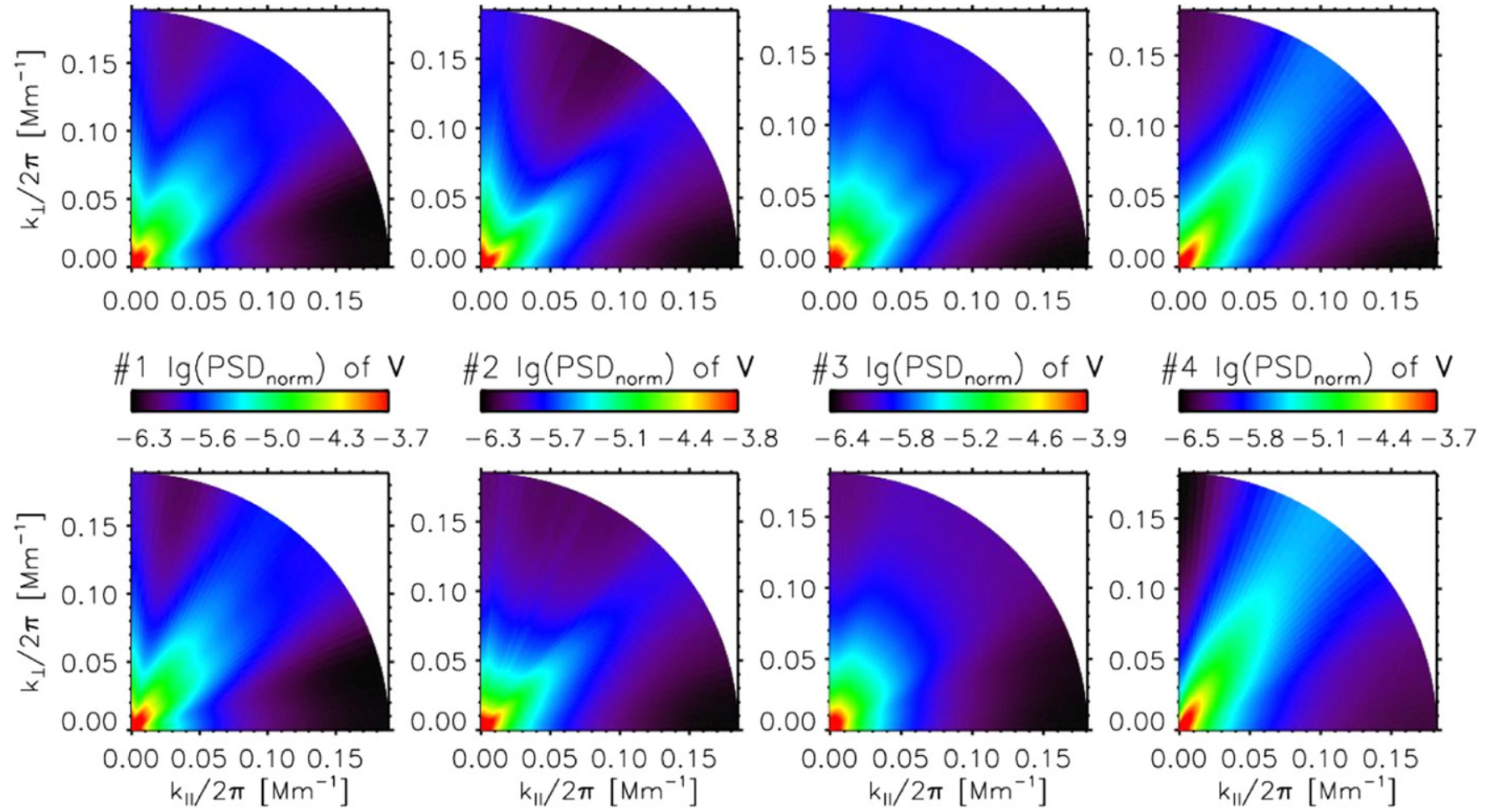

Figure 2. Spectra $\mathrm{PSD}_{2 \mathrm{D}}$ of $\boldsymbol{B}$ (upper panels) and $\boldsymbol{V}$ (lower panels) that are normalized to the maximum value for the four fast solar wind streams.

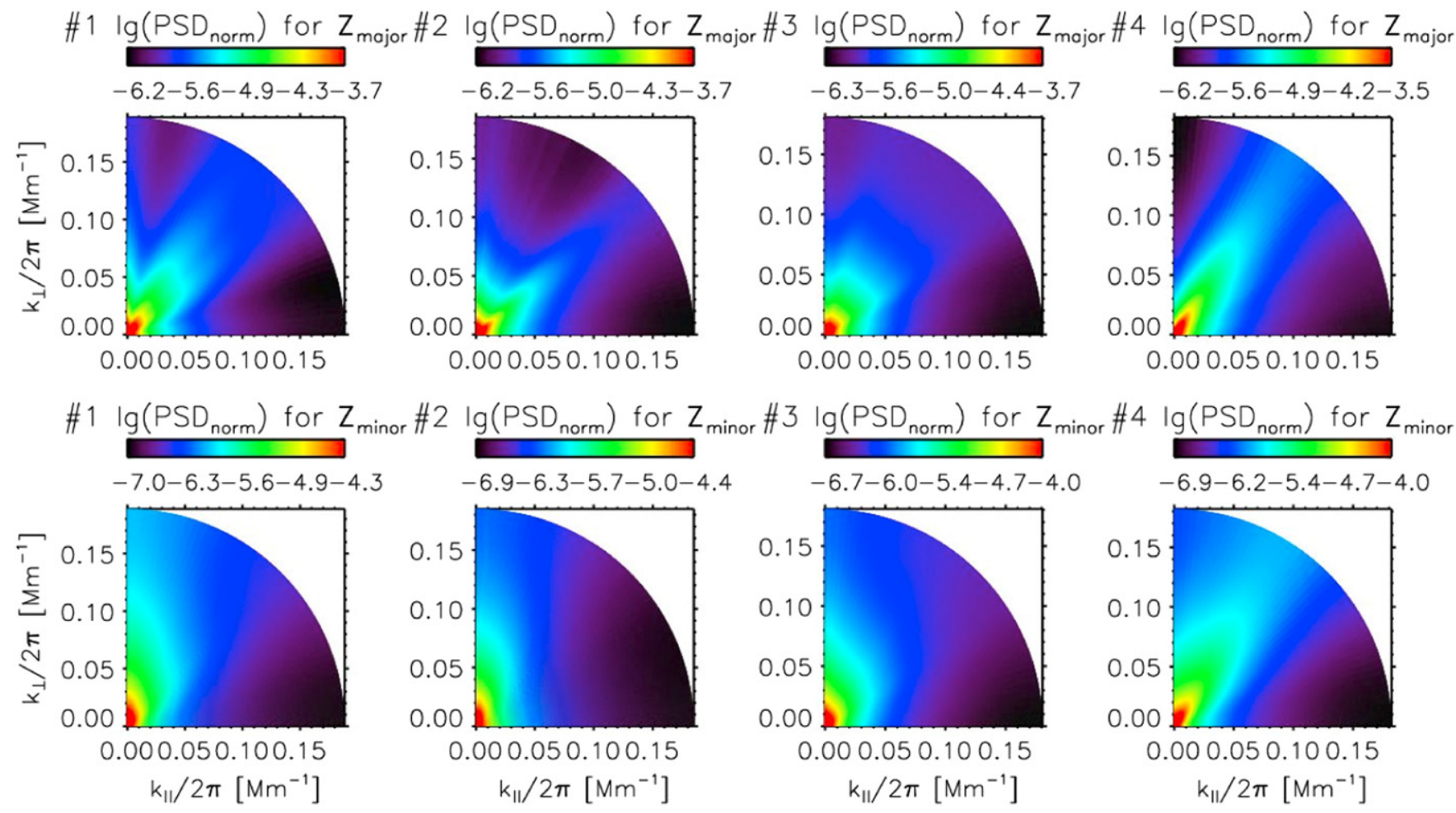

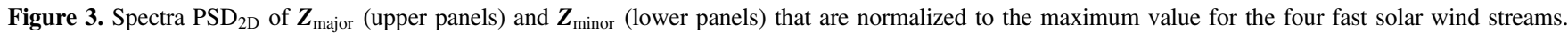



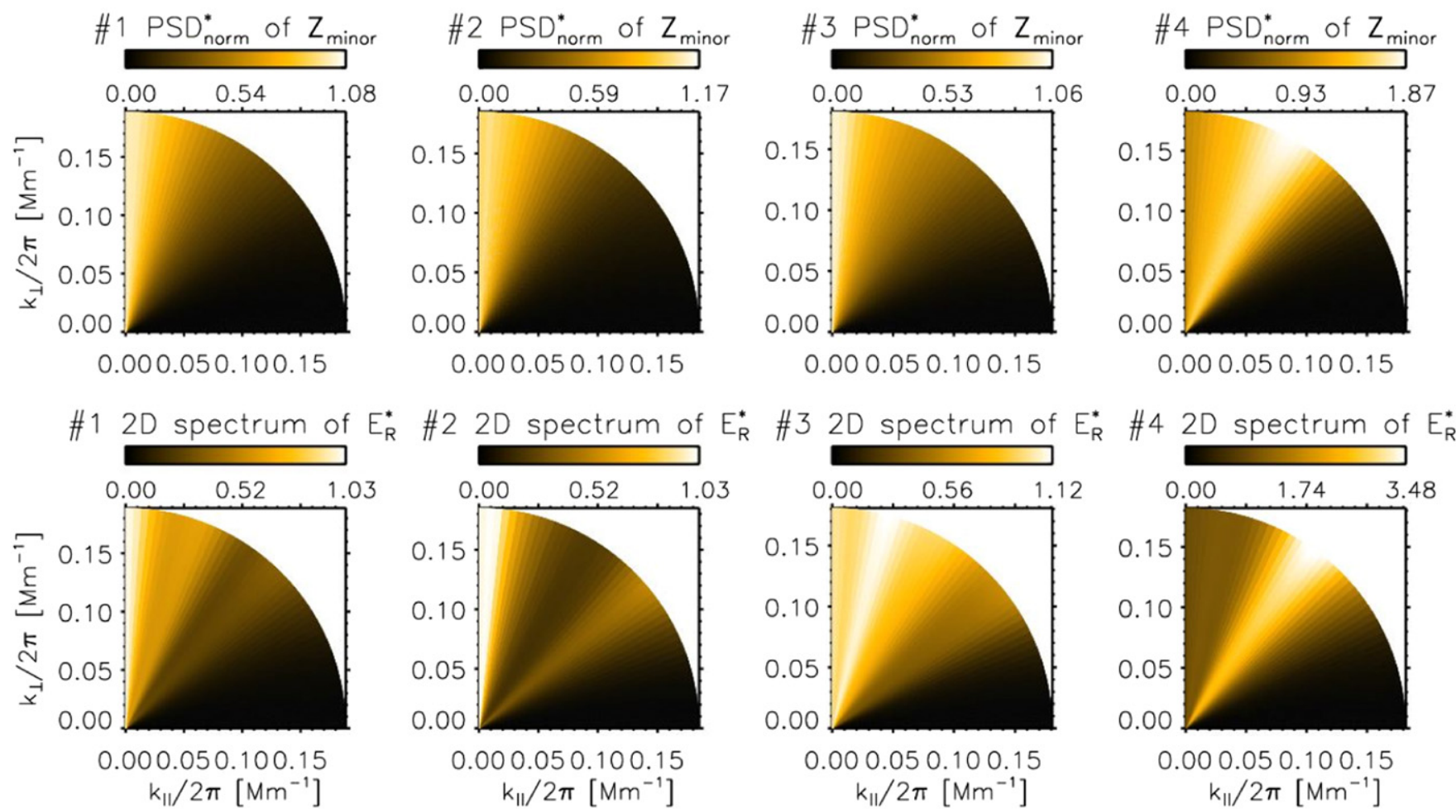

Figure 4. Normalized spectra of $\boldsymbol{Z}_{\text {minor }}\left(\frac{\boldsymbol{Z}_{\text {minor }}\left(k_{\|}, k_{\perp}\right)}{\boldsymbol{Z}_{\text {minor }}\left(k_{\|}=0, k_{\perp}\right)}\right.$; upper panels) and residual energy $\left(\frac{E_{\mathrm{R}}\left(k_{\|}, k_{\perp}\right)}{E_{\mathrm{R}}\left(k_{\|}=0, k_{\perp}\right)}\right.$; lower panels) for the four fast solar wind streams.

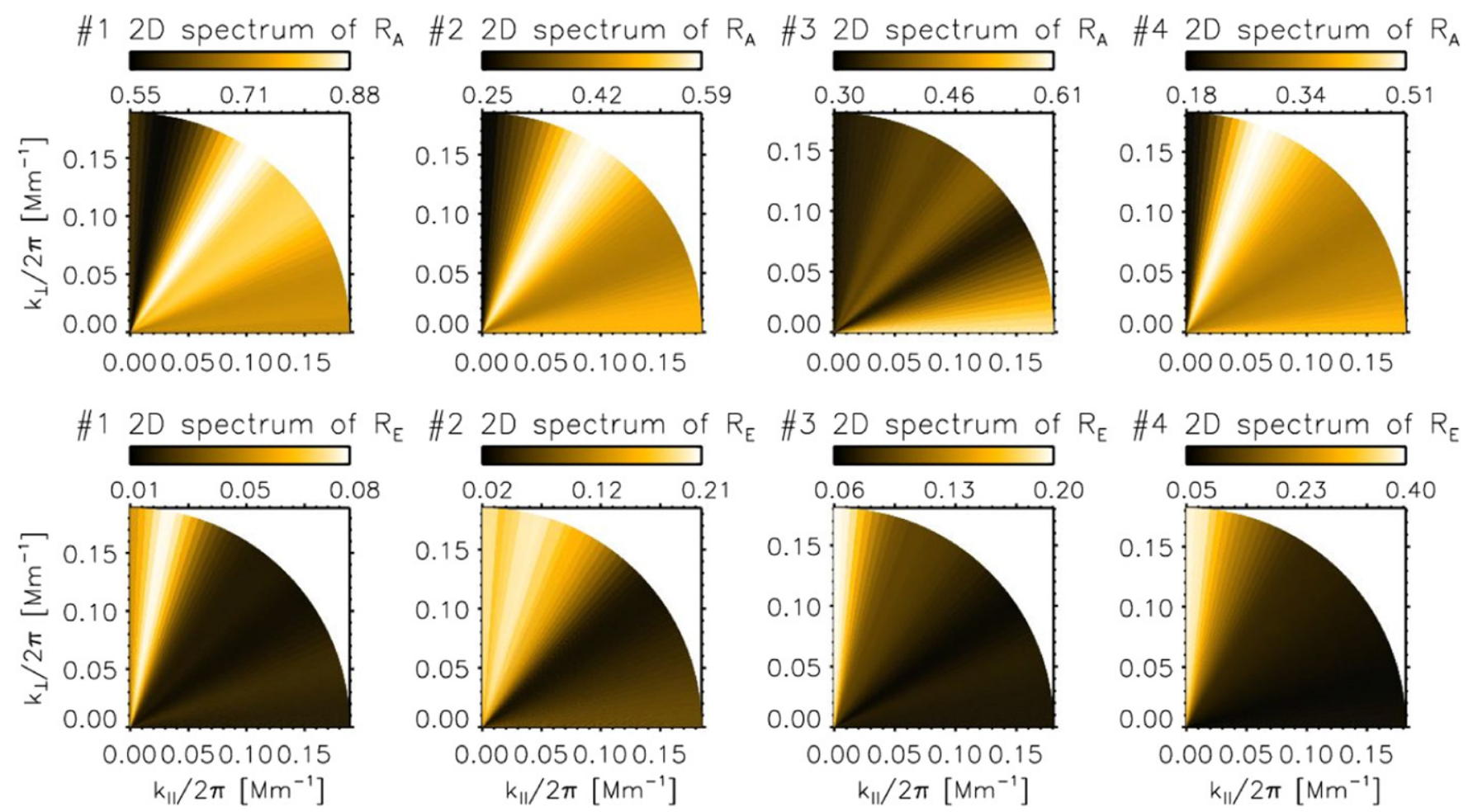

Figure 5. Wave-vector distribution of Alfvén ratio $R_{\mathrm{A}}$ (upper panels) and Elsässer ratio $R_{\mathrm{E}}$ (lower panels) for the four fast solar wind streams.

energy $E_{\mathrm{R}, \text { norm }}, E_{\mathrm{R}}$ is concentrated at small $k_{\|}$. This result gives the clear observational support to the simulation results of Boldyrev \& Perez (2009) and Wang et al. (2011), which showed a condensate of magnetic energy during the cascading of Alfvén waves due to the breakdown of the mirror symmetry in nonbalanced turbulence.

The distribution of $R_{\mathrm{A}}$ (upper panels in Figure 5) and $R_{\mathrm{E}}$ (lower panels in Figure 5) both show anisotropy. Close to the 
$k_{\perp}$ axis, $R_{\mathrm{A}}$ becomes smaller, suggesting that the dominance of magnetic energy over the kinetic energy becomes significant at small $k_{\|} . R_{\mathrm{E}}$ close to the $k_{\perp}$ axis is much larger than at other angles, suggesting that the difference between the energy of $\boldsymbol{Z}_{\text {major }}$ and that of $\boldsymbol{Z}_{\text {minor }}$ is larger close to the $k_{\perp}$ axis.

\section{SUMMARY AND DISCUSSIONS}

In this paper, we first did a benchmark test of the conversion between $\mathrm{CF}_{2 \mathrm{D}}$ and $\mathrm{PSD}_{2 \mathrm{D}}$, confirming that the conversion obtained directly from the corresponding formula, by the transformation (PSD tomography method) based on the projection-slice theorem, and by the transformation based on two-dimensional inverse Fourier transform are in accordance with each other. This experiment corroborates the applicability of the transformation (PSD tomography method) based on the projection-slice theorem to estimate $\mathrm{PSD}_{2 \mathrm{D}}$.

Based on the transformation, we investigated the spectral anisotropy of Elsässer variables in 2D wave-vector space for the first time. We also studied the distribution of residual energy $E_{\mathrm{R}}$, Alfvén ratio $R_{\mathrm{A}}$, and Elsässer ratio $R_{\mathrm{E}}$, which have not been studied in the $\left(k_{\|}, k_{\perp}\right)$ space before. Four fast streams observed by the WIND spacecraft were studied in this work.

The spectra $\mathrm{PSD}_{2 \mathrm{D}}$ of $\boldsymbol{Z}_{\text {major }}$ and $\boldsymbol{Z}_{\text {minor }}$ both show anisotropy in the wave-vector space. However, the anisotropic patterns of $\boldsymbol{Z}_{\text {major }}$ and $\boldsymbol{Z}_{\text {minor }}$ are different and the anisotropy of $\boldsymbol{Z}_{\text {minor }}$ seems stronger than that of $\boldsymbol{Z}_{\text {major }}$, which is consistent with the observational results from the reduced spectrum (Wicks et al. 2011) and the simulation result (Cho \& Lazarian 2014).

For each of the four fast streams, the spectra $\mathrm{PSD}_{2 \mathrm{D}}$ of $\boldsymbol{B}, \boldsymbol{V}$, and $\boldsymbol{Z}_{\text {major }}$ share an anisotropic pattern similar to that obtained by He et al. (2013). PSD $_{2 \mathrm{D}}$ spectra are mainly distributed along a ridge that is inclined toward the $k_{\perp}$ axis. This suggests that $\boldsymbol{Z}_{\text {major }}$ probably corresponds to oblique Alfvénic fluctuations propagating outward.

Differently from that of $\boldsymbol{B}, \boldsymbol{V}$, and $\boldsymbol{Z}_{\text {major }}$, the $\mathrm{PSD}_{2 \mathrm{D}}$ of $\boldsymbol{Z}_{\text {minor }}$ is distributed mainly along the $k_{\perp}$ axis. The Elsässer ratio $R_{\mathrm{E}}$ is larger at large $\theta_{k B}$ angles than at other angles, suggesting that the difference between the spectra $\mathrm{PSD}_{2 \mathrm{D}}$ of $\boldsymbol{Z}_{\text {major }}$ and that of $\boldsymbol{Z}_{\text {minor }}$ becomes more evident when it gets close to the $k_{\perp}$ axis. The spectra $\mathrm{PSD}_{2 \mathrm{D}}$ of $\boldsymbol{Z}_{\text {minor }}$ normalized to the $\mathrm{PSD}_{2 \mathrm{D}}$ with the same $k_{\perp}$ but with $k_{\|}=0$ further demonstrates that the power of $\boldsymbol{Z}_{\text {minor }}$ is concentrated at small $k_{\|}$. The Alfvén ratio $R_{\mathrm{A}}$ close to the $k_{\perp}$ axis is much smaller compared to that at other angles. If the plasma is thermally anisotropic and component-drifted, the Alfvén ratio will be very low, even when $\boldsymbol{Z}_{\text {minor }}$ stands for the inward propagating Alfvén wave. So, the presence of inward propagating Alfvén waves can not be excluded. If the cascade of $\boldsymbol{Z}_{\text {minor }}$ is driven by $\boldsymbol{Z}_{\text {major }}$, this may suggest that the cascade is anisotropic and probably mainly along the $k_{\perp}$ direction. The magnetic structure without velocity fluctuations and the nonAlfvénic fluctuation with $k_{\|}=0$ both could lead to the power concentration of $\boldsymbol{Z}_{\text {minor }}$ and the low Alfvén ratio. Further work is required in the future to understand what $\boldsymbol{Z}_{\text {minor }}$ mostly represents.

Though the spectra of $\boldsymbol{B}$ and $\boldsymbol{V}$ share a similar spectral anisotropic pattern, there are still differences between them as revealed by the anisotropic distribution of $E_{\mathrm{R}}$ and $R_{\mathrm{A}}$. Close to the $k_{\perp}$ axis, $R_{\mathrm{A}}$ becomes smaller and $\left|E_{\mathrm{R}}\right|$ becomes larger, suggesting that the dominance of the magnetic energy over the kinetic energy becomes significant. The residual energy condensate at small $k_{\|}$confirms observationally the findings in the simulation results of Boldyrev \& Perez (2009) and Wang et al. (2011).

It should be noted that $\boldsymbol{Z}_{\text {minor }}$ may be anti-correlated with $\boldsymbol{Z}_{\text {major }}$ due to the dominance of magnetic energy over kinetic energy. The unequipartition between magnetic and kinetic energy may be the case for Alfvén waves with kinetic effects if the plasma is thermally anisotropic and component-drifted. We have tried to re-estimate the spectra $\mathrm{PSD}_{2 \mathrm{D}}$ of $\boldsymbol{Z}_{\text {major }}$ and $\boldsymbol{Z}_{\text {minor }}$ after correcting for kinetic effects from the thermal anisotropy. The recalculated distribution of $\mathrm{PSD}_{2 \mathrm{D}}$ of $\boldsymbol{Z}_{\text {major }}$ remain almost unchanged. However, the $\mathrm{PSD}_{2 \mathrm{D}}$ of $\boldsymbol{Z}_{\text {minor }}$ after correcting the thermal anisotropy cannot be reconstructed with good quality, which might be due to the possible over-correction of the thermal anisotropy on the weak signal of $\boldsymbol{Z}_{\text {minor }}$.

In the critical balance theory of Goldreich \& Sridhar (1995), the eddies are filament shaped. In simulations, the eddies usually have a ribbon shape (Biskamp 2000; Müller \& Biskamp 2000; Maron \& Goldreich 2001) rather than a filament. Boldyrev (2006) extended critical balance theory to account for this 3D anisotropy. Chen et al. (2012) investigated the local three-dimensional structure functions of the inertial range plasma turbulence based on observation for the first time. They found that the Alfvénic fluctuations are three-dimensionally anisotropic dependent on the scales. Recently, the spectral properties has been investigated in 3D MHD simulations by Dong et al. (2014) and Verdini \& Grappin (2015). They found many spectral anisotropy properties, including the 3D structure functions anisotropy as reported by Chen et al. (2012), could be explained in part by the solar wind expansion, which would lead to different fluctuation levels in the different magnetic field components. In the future, we intend to extend this method to three dimensions to investigate the PSD in 3D wave-vector space and compare the result with former theoretical and simulation results. To promote the usage of this method, it is necessary to conduct further calibrations of this procedure on numerical data of turbulence by comparing the reconstructed PSD with the known PSD (Oughton et al. 2015).

The group from Peking University is supported by NSFC under 41174148, 41222032, 41231069, 41421003, 41474147, 41274172, and 41474148. C.H.K.C. is supported by an Imperial College Junior Research Fellowship. J.S.H., C.Y.T., C.H.K.C., X.W., and R.W. are also members of the ISSI/ISSIBJ international team 304.

\section{REFERENCES}

Bavassano, B., Pietropaolo, E., \& Bruno, R. 1998, JGR, 103, 6521

Belcher, J. W., \& Davis, L., Jr. 1971, JGR, 76, 3534

Biskamp, D., \& Müller, W.-C. 2000, PhPl, 7, 4889

Boldyrev, S. 2006, PhRvL, 96, 115002

Boldyrev, S., \& Perez, J. C. 2009, PhRvL, 103, 225001

Boldyrev, S., Perez, J. C., Borovsky, J. E., \& Podesta, J. J. 2011, ApJL, 741, L19

Boldyrev, S., Perez, J. C., \& Zhdankin, V. 2012, in AIP Conf. Ser. 1436, Physics of the Heliosphere: A 10 Year Retrospective, ed. J. Heerikhuisen et al. (Melville, NY: AIP), 18

Bruno, R., Bavassano, B., \& Pietropaolo, E. 1996, in AIP Conf. Ser. 382, Proceedings of the Eighth International Solar Wind Conference, ed. D. Winterhalter et al. (Melville, NY: AIP), 229

Chen, C. H. K., Bale, S. D., Salem, C. S., \& Maruca, B. A. 2013, ApJ, 770, 125 Chen, C. H. K., Horbury, T. S., Schekochihin, A. A., et al. 2010, PhRvL, 104, 255002

Chen, C. H. K., Mallet, A., Schekochihin, A. A., et al. 2012, ApJ, 758, 120

Chen, C. H. K., Mallet, A., Yousef, T. A., Schekochihin, A. A., \& Horbury, T. S. 2011, MNRAS, 415, 3219 
Cho, J., \& Lazarian, A. 2014, ApJ, 780, 30

Dong, Y., Verdini, A., \& Grappin, R. 2014, ApJ, 793, 118

Franci, L., Verdini, A., Matteini, L., Landi, S., \& Hellinger, P. 2015, ApJL, 804, L39

Galtier, S., Nazarenko, S. V., Newell, A. C., \& Pouquet, A. 2000, JPIPh, 63, 447

Gogoberidze, G., Chapman, S. C., \& Hnat, B. 2012, PhPl, 19, 102310

Goldreich, P., \& Sridhar, S. 1995, ApJ, 438, 763

Grappin, R., Leorat, J., \& Pouquet, A. 1983, A\&A, 126, 51

He, J., Marsch, E., Tu, C., Yao, S., \& Tian, H. 2011, ApJ, 731, 85

He, J., Tu, C., Marsch, E., Bourouaine, S., \& Pei, Z. 2013, ApJ, 773, 72

Horaites, K., Boldyrev, S., Krasheninnikov, S. I., et al. 2015, PhRvL, 114, 245003

Horbury, T. S., Forman, M., \& Oughton, S. 2008, PhRvL, 101, 175005

Horbury, T. S., Wicks, R. T., \& Chen, C. H. K. 2012, SSRv, 172, 325

Howes, G. G., \& Nielson, K. D. 2013, PhPl, 20, 072302

Lepping, R. P., Acuna, M. H., Burlaga, L. F., et al. 1995, SSRv, 71, 207

Lin, R. P., Anderson, K. A., Ashford, S., et al. 1995, SSRv, 71, 125

Lithwick, Y., \& Goldreich, P. 2003, ApJ, 582, 1220

Lithwick, Y., Goldreich, P., \& Sridhar, S. 2007, ApJ, 655, 269

Luo, Q. Y., \& Wu, D. J. 2010, ApJL, 714, L138

Maron, J., \& Goldreich, P. 2001, ApJ, 554, 1175

Matteini, L., Horbury, T. S., Neugebauer, M., \& Goldstein, B. E. 2014, GeoRL, 41, 259

Matthaeus, W. H., \& Goldstein, M. L. 1982, JGR, 87, 6011
Matthaeus, W. H., Servidio, S., Dmitruk, P., et al. 2012, ApJ, 750, 103

Müller, W.-C., \& Biskamp, D. 2000, PhRvL, 84, 475

Müller, W.-C., \& Grappin, R. 2005, PhRvL, 95, 114502

Narita, Y., Glassmeier, K.-H., Sahraoui, F., \& Goldstein, M. L. 2010, PhRvL, 104, 171101

Nielson, K. D., Howes, G. G., \& Dorland, W. 2013, PhPl, 20, 072303

Oughton, S., Matthaeus, W. H., Wan, M., \& Osman, K. T. 2015, RSPTA, 373, 20140152

Perez, J. C., \& Boldyrev, S. 2009, PhRvL, 102, 025003

Podesta, J. J. 2009, ApJ, 698, 986

Roberts, D. A., Klein, L. W., Goldstein, M. L., \& Matthaeus, W. H. 1987, JGR, 92, 11021

Sahraoui, F., Goldstein, M. L., Belmont, G., Canu, P., \& Rezeau, L. 2010, PhRvL, 105, 131101

Shebalin, J. V., Matthaeus, W. H., \& Montgomery, D. 1983, JPIPh, 29, 525

Tu, C. Y., \& Marsch, E. 1992, in Solar Wind Seven, Proceedings of the 3rd COSPAR Colloquium, ed. E. Marsch \& R. Schwenn (Goslar, Germany), 549

Tu, C.-Y., \& Marsch, E. 1993, JGR, 98, 1257

Verdini, A., \& Grappin, R. 2015, ApJL, 808, L34

Wang, X., Tu, C., He, J., Marsch, E., \& Wang, L. 2014, ApJL, 783, L9

Wang, Y., Boldyrev, S., \& Perez, J. C. 2011, ApJL, 740, L36

Wicks, R. T., Horbury, T. S., Chen, C. H. K., \& Schekochihin, A. A. 2011, PhRvL, 106, 045001 